\title{
Perfil clínico-epidemiológico de mulheres em tratamento oncológico de carcinoma ductal invasivo da mama
}

Clinical-epidemiological profile of women undergoing oncological treatment for invasive ductal breast carcinoma

Perfil clínico-epidemiológico de las mujeres en tratamiento oncológico por carcinoma ductal invasivo de mama

Recebido: 09/01/2021 | Revisado: 11/01/2021 | Aceito: 16/01/2021 | Publicado: 19/01/2021

Simone Yuriko Kameo

ORCID: https://orcid.org/0000-0002-0035-2415 Universidade Federal de Sergipe, Brasil E-mail: simonekameo@hotmail.com

Ricardo Barbosa Lima

ORCID: https://orcid.org/0000-0001-5274-4800

Universidade de São Paulo, Brasil

E-mail: ricardobarbosalima@usp.br

Maria Júlia Oliveira Ramos

ORCID: https://orcid.org/0000-0003-4729-4416 Universidade Federal de Sergipe, Brasil

E-mail: maju.oramos@gmail.com.

Tiago Vasconcelos Fonseca

ORCID: https://orcid.org/0000-0002-7632-9860 Universidade Federal de Sergipe, Brasil

E-mail: tiagovasconfonseca@gmail.com

Andressa Cabral Vassilievitch

ORCID: https://orcid.org/0000-0002-2421-4607

Universidade Federal de Sergipe, Brasil

E-mail: andressavassi98@gmail.com

Jéssica dos Santos Costa

ORCID: https://orcid.org/0000-0003-2404-6604 Instituto de Medicina Integral Professor Fernando Figueira, Brasil

E-mail: jessicas_costa@outlook.com

José Cleyton de Oliveira Santos

ORCID: https://orcid.org/0000-0001-5616-7625 Universidade Federal de Sergipe, Brasil

E-mail: cleyton-121@hotmail.com

Dayane Ketlyn da Cunha Santos

ORCID: https://orcid.org/0000-0002-9312-4891 Universidade Federal de Sergipe, Brasil

E-mail: daayketlyn27@gmail.com

Bruno Ferreira Amorim

ORCID: https://orcid.org/0000-0002-2581-2983 Universidade Federal de Sergipe, Brasil

E-mail: brunofamorim@hotmail.com.br

Pabliane Matias Lordelo Marinho ORCID: https://orcid.org/0000-0001-6190-0844 Universidade de São Paulo, Brasil E-mail: marinho.pabliane@gmail.com

Namie Okino Sawada

ORCID: https://orcid.org/0000-0002-1874-3481

Universidade Federal de Alfenas, Brasil

E-mail: namie.sawada@unifal-mg.edu.br

Glebson Moura Silva

ORCID: https://orcid.org/0000-0002-4977-2787

Universidade Federal de Sergipe, Brasil

E-mail: glebsonmoura@yahoo.com.br

\section{Resumo}

O objetivo do estudo foi descrever o perfil clínico-epidemiológico de mulheres em tratamento oncológico para carcinoma ductal invasivo da mama. Trata-se de um estudo transversal, descritivo e quantitativo por meio da coleta de 
dados em prontuários clínicos de mulheres em tratamento oncológico do câncer de mama em um serviço privado de oncologia, considerando variáveis sociodemográficas e clínicas. Foram identificadas 125 mulheres que se adequaram aos critérios de inclusão propostos. A maioria das pacientes incluídas eram sergipanas (71,2\%), pardas ou negras $(65,6 \%)$, com companheiro $(56 \%)$ e com média de idade de 53,5 $\pm 11,4$ anos. Destas, $80,8 \%$ não possuíam ensino superior e 77,6\% possuíam renda familiar entre um e três salários mínimos. Todas se submeteram à quimioterapia, $64,8 \%$ à mastectomia total e $36 \%$ à radioterapia. Além disso, 32,8\% apresentaram mudanças na autoimagem, 24,8\% na autoestima, $64,8 \%$ sinais de ansiedade e 49,6\% sintomas depressivos. Além disso, $40 \%$ eram portadoras de hipertensão arterial sistêmica. O perfil clínico-epidemiológico das mulheres com carcinoma ductal invasivo da mama descrito é amplo e possui componentes sociodemográficos importantes. O tratamento oncológico pode desencadear alterações clínicas relevantes para a saúde, bem-estar e qualidade de vida destas pacientes.

Palavras-chave: Epidemiologia; Neoplasias da mama; Oncologia; Saúde da mulher.

\begin{abstract}
The aim of this study was to describe the clinical-epidemiological profile of women undergoing oncological treatment for invasive ductal breast carcinoma. This is a cross-sectional, descriptive and quantitative study carried out by data collection from clinical records of women undergoing breast cancer treatment in a private oncology service, considering sociodemographic and clinical variables. 125 women who met the proposed inclusion criteria were identified. Most of the patients were from Sergipe $(71,2 \%)$, black or mixed $(65,6 \%)$, with a partner $(56 \%)$ and with a mean age of 53,5 $\pm 11,4$ years. Of these, $80,8 \%$ had no higher education and $77,6 \%$ had family income between one and three minimum wages. All patients underwent chemotherapy, 64,8\% radical mastectomy and 36\% radiotherapy. In addition, 32,8\% showed changes in self-image, $24,8 \%$ in self-esteem, $64,8 \%$ signs of anxiety and 49,6\% depressive symptoms. In addition, $40 \%$ had systemic arterial hypertension. The clinical-epidemiological profile of women with invasive ductal breast carcinoma described is wide and has important sociodemographic components. Cancer treatment can trigger clinical changes relevant to the health, well-being and quality of life of these patients.
\end{abstract}

Keywords: Breast neoplasms; Epidemiology; Oncology; Women's health.

\title{
Resumen
}

El objetivo de este estudio fue describir el perfil clínico-epidemiológico de mujeres en tratamiento oncológico por carcinoma ductal invasivo de mama. Se trata de un estudio transversal, descriptivo y cuantitativo mediante la recolección de datos de historias clínicas de mujeres en tratamiento de cáncer de mama en un servicio oncológico privado, considerando variables sociodemográficas y clínicas. Se identificaron 125 mujeres que cumplían con los criterios de inclusión propuestos. La mayoría de los pacientes incluidos eran de Sergipe (71,2\%), morenos o negros $(65,6 \%)$, con pareja (56\%) y con una edad media de 53,5 $\pm 11,4$ años. De estos, el 80,8\% no tenía estudios superiores y el 77,6\% tenía ingresos familiares entre uno y tres salarios mínimos. Todos fueron sometidos a quimioterapia, 64,8\% a mastectomía total y $36 \%$ a radioterapia. Además, el 32,8\% presentó alteraciones en la autoimagen, el 24,8\% en la autoestima, el 64,8\% signos de ansiedad y el 49,6\% síntomas depresivos. Además, el $40 \%$ tenía hipertensión arterial sistémica. El perfil clínico y epidemiológico de las mujeres con carcinoma ductal invasivo de mama descrito es amplio y tiene importantes componentes sociodemográficos. El tratamiento del cáncer puede desencadenar cambios clínicos relevantes para la salud, el bienestar y la calidad de vida de estos pacientes.

Palabras clave: Epidemiología; La salud de la mujer; Neoplasias de mama; Oncología.

\section{Introdução}

As neoplasias mamárias consistem em doenças com alto grau de letalidade para mulheres e uma das principais causas de mortalidade desta população, sendo responsável por até $23 \%$ das mortes por câncer no mundo. O risco do câncer de mama aumenta com o avanço da idade, de modo que após os 50 anos a incidência desta neoplasia é de dois novos casos para 1.000 mulheres (2:1.000), bem como espera-se que em 2021 a incidência alcance a marca de 85 novos casos a cada 100.000 mulheres (Yeo \& Guan; 2017; Akram et al; 2017).

O câncer de mama se configura como uma neoplasia de grande variação histopatológica e molecular, sendo o tipo de câncer mais comum em mulheres. Caracterizações de natureza epidemiológica e clínica de tumores mamários permitem compreender as particularidades das diversas apresentações nas populações ao redor do mundo, promovendo subsídios para abordagens individualizadas em diversas comunidades, visando a prevenção e o diagnóstico precoce deste problema de saúde pública (Yeo \& Guan, 2017; Akram et al., 2017).

Dentre os diversos subtipos histopatológicos, o carcinoma ductal invasivo da mama é o mais comum. Trata-se de uma 
variante infiltrativa na qual as células neoplásicas se proliferam além da camada basal dos ductos mamários, podendo disseminar para outros locais do corpo. Diversos fatores estão relacionados ao desenvolvimento do câncer de mama, incluindo componentes genéticos (BRCA1 e BRCA2), biológicos (hormônios esteroidais e contraceptivos orais) e ambientais (estilo de vida, envelhecimento e hábitos) (Sun et al., 2017). No Brasil, assim como em outros países em desenvolvimento, apesar do aumento das ações preventivas e incorporação de novas evidências e tecnologias no diagnóstico e tratamento do câncer de mama, houve o aumento da incidência desta neoplasia nos últimos anos (Cabral et al., 2019).

O acesso ao diagnóstico precoce e o intervalo de tempo entre o diagnóstico e o início do tratamento têm impacto direto na sobrevida de mulheres com câncer de mama. A oferta de serviços de saúde é primordial para alcançar melhores índices relacionados a esta neoplasia, entretanto, fatores sociodemográficos como baixo nível socioeconômico, etnia, escolaridade e iniquidades em saúde interferem diretamente nos desfechos relacionados ao câncer de mama. Estes fatores ainda contribuem para o surgimento de comorbidades que pioram o prognóstico, como resultado da dificuldade em acessar serviços de saúde de forma adequada em todas as suas dimensões, experiência frequente em populações socioeconomicamente vulneráveis de países em desenvolvimento (Cabral et al., 2019; Mathew et al., 2019).

Além destes componentes, os dados epidemiológicos relacionados ao câncer de mama estão fortemente associados a características clínicas que abrangem desde o diagnóstico histopatológico/molecular até os tratamentos empregados (Anampa et al., 2015). Com o avanço das evidências, o arsenal de opções terapêuticas para o câncer de mama evoluiu. Atualmente, o tratamento de neoplasias mamárias pode envolver quimioterapia, radioterapia e cirurgias, combinadas entre si ou não. A terapia sistêmica adjuvante reduz o tamanho do tumor e a indicação de mastectomias e abordagens radioterápicas estão associadas à redução das recorrências locais (Moo et al., 2018).

Dentre as possíveis apresentações clínicas, considerando os carcinomas invasivos, o subtipo ductal é o mais prevalente em diversos estudos, variando entre 50-80\% (Moo et al., 2018; Arps et al., 2013), sendo uma progressão clínica do carcinoma ductal in situ em 10-30\% das pacientes (Casasent et al., 2018). Em estudos histopatológicos, o carcinoma ductal invasivo frequentemente é utilizado como referência nas análises (Li., et al 2005), demonstrando a importância de compreender e caracterizar esta apresentação. Sendo assim, diante deste contexto, o objetivo deste estudo é descrever o perfil clínicoepidemiológico de mulheres em tratamento oncológico de carcinoma ductal invasivo da mama.

\section{Metodologia}

Trata-se de um estudo transversal, quantitativo e descritivo desenvolvido por meio da coleta de dados em prontuários clínicos das participantes. A execução deste estudo foi baseada nas diretrizes nacionais de bioética em pesquisa com seres humanos e obteve a apreciação e aprovação pelo Comitê de Ética em Pesquisa da Escola de Enfermagem de Ribeirão Preto da Universidade de São Paulo (CEP-EERP/USP), sob parecer de número 531.146 (CAAE: 20834513.0.0000.5393). Os procedimentos metodológicos foram executados com rigor científico, bem como os princípios bioéticos e as diretrizes propostas na resolução 466 de 2012 do Conselho Nacional de Saúde, assegurando a confidencialidade dos dados obtidos nos questionários e prontuários clínicos acessados.

O local de pesquisa foi um ambulatório privado de oncologia situado em um município do estado de Sergipe, sem ligação com o Sistema Único de Saúde (SUS). A escolha do campo de pesquisa foi tomada com base no fluxo de pacientes em tratamento oncológico por meio de diversas modalidades, fornecendo uma população significativa para o objetivo de pesquisa. A coleta dos dados ocorreu entre fevereiro de 2014 e fevereiro de 2015.

Inicialmente, 560 prontuários clínicos foram identificados no serviço no início da coleta dos dados. Este volume inicial foi triado com base nos critérios de inclusão para determinar a amostra por duas pesquisadoras da área de oncologia. Foram incluídos os prontuários clínicos mulheres maiores de 18 anos, que apresentaram diagnóstico citopatológico de carcinoma ductal 
invasivo da mama (verificado no prontuário) e que estavam em tratamento durante o período de coleta de dados. Cada prontuário foi analisado em um único momento, sem acompanhamento durante o período da coleta.

No que se refere aos prontuários clínicos, considerando que se tratavam de prontuários tradicionais/não-eletrônicos, foram incluídos apenas os que puderam ser localizados e estavam preenchidos de forma completa e legível pelos profissionais do serviço de oncologia estudado. A ausência de dados ou a adequação parcial aos critérios levaram à exclusão das pacientes e seus respectivos prontuários clínicos. O Termo de Consentimento Livre e Esclarecido (TCLE) foi dispensado pela natureza documental do estudo.

As variáveis sociodemográficas investigadas e coletadas dos prontuários clínicos foram: idade, moradia, zona (rural ou urbana), rede de abastecimento de água, saneamento, cor da pele, estado civil, presença/ausência de companheiro, quantidade de filhos, escolaridade, ocupação, situação ocupacional, fonte de renda, renda familiar e religião. As variáveis clínicas de interesse foram: diagnóstico citopatológico, tempo de diagnóstico, extensão da neoplasia, comorbidades, procedimentos cirúrgicos realizados, radioterapia, quimioterapia e quimiorradioterapia, além dos protocolos antineoplásicos adotados e dos sinais e sintomas associados ao tratamento oncológico.

As pesquisadoras coletaram de forma independente os dados clínicos utilizando um formulário próprio para registro. Após a coleta, os dados obtidos foram cruzados para reduzir possíveis falhas e divergências anteriormente à tabulação em planilha pela ferramenta Planilhas Google® para posterior análise e interpretação. Foram operadas estatísticas descritivas em frequências absolutas e relativas das variáveis, identificando a ocorrência das mesmas na população estudada.

\section{Resultados e Discussão}

Foram identificadas 125 mulheres $(n=125)$ em tratamento oncológico de carcinoma ductal invasivo da mama que se adequaram aos critérios de inclusão propostos, representando $22,3 \%$ de todos os pacientes do serviço de oncologia estudado. A média de idade da amostra foi 53,5 $\pm 11,4$ anos, variando entre 26 e 83 anos. A Tabela 1 apresenta os dados sociodemográficos e econômicos da amostra. A Tabela 2 apresenta os dados clínicos coletados da amostra.

Tabela 1. Características sociodemográficas das pacientes em tratamento oncológico de carcinoma ductal invasivo - Aracaju, Sergipe, Brasil (2015).

\begin{tabular}{|c|c|c|}
\hline Variáveis & $\begin{array}{l}\text { Frequência absoluta } \\
\text { (n) }\end{array}$ & $\begin{array}{c}\text { Frequência relativa } \\
(\%)\end{array}$ \\
\hline \multicolumn{3}{|l|}{ Residência/Moradia } \\
\hline Sergipe & 89 & 71,2 \\
\hline Bahia & 17 & 13,6 \\
\hline Alagoas & 7 & 5,6 \\
\hline Outros & 12 & 9,6 \\
\hline Zona urbana & 100 & 80,0 \\
\hline Zona rural & 25 & 20,0 \\
\hline Casa própria & 103 & 82,4 \\
\hline Casa alugada & 13 & 10,4 \\
\hline Outros & 9 & 7,2 \\
\hline Com acesso à rede de água e esgoto & 90 & 72,0 \\
\hline Sem acesso à rede de água e esgoto & 35 & 28,0 \\
\hline \multicolumn{3}{|l|}{ Etnia } \\
\hline Branca & 43 & 34,4 \\
\hline & 4 & \\
\hline
\end{tabular}


Parda

Negra

Estado civil

\begin{tabular}{l}
\hline Solteira \\
Casada \\
União consensual \\
Viúva \\
Separada \\
Divorciada \\
\hline Companheiro(a)
\end{tabular}

Sem companheiro(a)

Quantidade de filhos

Escolaridade

Não escolarizada

Ensino fundamental

Ensino médio 36,0

Ensino superior

\section{Ocupação}

Lavradora

Do lar

Professora

Auxiliar administrativa

Diarista/doméstica

10

5,6

Costureira

Auxiliar de serviços gerais

5,6

Vendedora

\begin{tabular}{l}
\hline Situação ocupacional \\
\hline Desempregada \\
Empregada \\
Aposentada \\
Autônoma \\
Licença \\
\hline Fonte de renda \\
\hline
\end{tabular}

Não declararam fonte de renda

Trabalho

Aposentadoria ou pensão

Doações e auxílio de familiares 


\begin{tabular}{lcc}
\hline$<1$ salário mínimo & 7 & 5,6 \\
$\geq 1$ e $\leq 3$ salários mínimos & 97 & 77,6 \\
$\geq 4$ salários mínimos & 21 & 16,8 \\
\hline Religião & & 74,4 \\
\hline Católica & 93 & 16,8 \\
Evangélica & 21 & 8,8 \\
Outras & 11 & 92,0 \\
Praticante & 115 & 8,0 \\
Não praticante & 10 & 100,0 \\
\hline Crença em ser superior & & \\
\hline Sim & 125 &
\end{tabular}

Fonte: Autores.

Tabela 2. Perfil clínico das pacientes em tratamento oncológico de carcinoma ductal invasivo - Aracaju, Sergipe, Brasil (2015).

\begin{tabular}{|c|c|c|}
\hline Variáveis & $\begin{array}{c}\text { Frequência absoluta } \\
(\text { (n) }\end{array}$ & $\begin{array}{c}\text { Frequência relativa } \\
(\%)\end{array}$ \\
\hline \multicolumn{3}{|l|}{ Tempo diagnóstico do câncer de mama } \\
\hline$<6$ meses & 6 & 4,8 \\
\hline$\geq 6$ meses e $\leq 12$ meses & 46 & 36,8 \\
\hline$>12$ meses & 73 & 58,4 \\
\hline \multicolumn{3}{|l|}{ Localização } \\
\hline Unilateral & 125 & 100,0 \\
\hline Bilateral & 0 & 0,0 \\
\hline \multicolumn{3}{|l|}{ Extensão da neoplasia da mama } \\
\hline Tumor local & 3 & 2,4 \\
\hline Disseminação local & 96 & 76,8 \\
\hline Disseminação distal & 26 & 20,8 \\
\hline \multicolumn{3}{|l|}{ Procedimentos cirúrgicos } \\
\hline Apenas biópsia & 17 & 13,6 \\
\hline Biópsia e remoção parcial da mama & 27 & 21,6 \\
\hline Biópsia e remoção total da mama & 81 & 64,8 \\
\hline \multicolumn{3}{|l|}{ Radioterapia } \\
\hline Nenhuma sessão & 80 & 64,0 \\
\hline$\leq 10$ sessões & 3 & 2,4 \\
\hline$>10$ sessões e $\leq 20$ sessões & 2 & 1,6 \\
\hline$>20$ sessões e $\leq 30$ sessões & 34 & 27,2 \\
\hline$>30$ sessões & 6 & 4,8 \\
\hline \multicolumn{3}{|l|}{ Quimioterapia } \\
\hline Sim & 125 & 100,0 \\
\hline \multicolumn{3}{|l|}{ Quimiorradioterapia } \\
\hline Sim & 45 & 36,0 \\
\hline Não & 80 & 64,0 \\
\hline
\end{tabular}




\section{Protocolos e agentes quimioterápicos}

Protocolo AC

doxorrubicina + ciclofosfamida

Protocolo AC-T

doxorrubicina + ciclofosfamida

seguido por paclitaxel ou docetaxel

Protocolo CMF

ciclofosfamida + metotrexato +5 -

fluorouracil

Protocolo FAC

ciclofosfamida + doxorrubicina +5 -

fluorouracil

Protocolo TAC

docetaxel + doxorrubicina + ciclofosfamida

Protocolo TC

docetaxel + ciclofosfamida

\begin{tabular}{l}
\hline Anastrozol \\
\hline Tamoxifeno \\
\hline Trastuzumabe \\
\hline $\begin{array}{l}\text { Sinais e sintomas associados ao } \\
\text { tratamento oncológico }\end{array}$ \\
\hline
\end{tabular}

Dor

Fadiga

Sinais de ansiedade

Sintomas depressivos

Náuseas e vômitos

Alterações na autoimagem

Alterações na autoestima

Sinais e sintomas simultâneos associados ao tratamento oncológico

Nenhum

1 ou 2 sinais ou sintomas

3 ou 4 sinais ou sintomas

5 ou 6 sinais ou sintomas

7 ou mais sinais ou sintomas

Comorbidades

\begin{tabular}{lcc}
\hline Hipertensão arterial sistêmica (HAS) & 50 & 40,0 \\
Artrite/artrose (AA) & 23 & 18,4 \\
Osteoporose/osteopenia (OO) & 15 & 12,0 \\
Diabetes mellitus (DM) & 14 & 11,2 \\
Dislipidemia & 9 & 7,2 \\
\hline HAS + DM & 12 & 9,6 \\
\hline AA + OO & 9 & 7,2 \\
\hline
\end{tabular}

Fonte: Autores.

Em nossos resultados, $65,6 \%$ das mulheres com carcinoma ductal invasivo da mama eram pretas ou pardas. Evidências recentes verificam disparidades raciais na epidemiologia do câncer de mama. Embora os valores de incidência de neoplasias mamárias em mulheres brancas sejam maiores em diversos países, mulheres pretas podem ser mais propensas aos desfechos 
negativos relacionados ao câncer, especialmente a mortalidade em todas as faixas etárias. Além disso, a incidência de câncer de mama antes dos 45 anos é maior em mulheres pretas, embora entre 60 e 84 anos seja maior em mulheres brancas. Por outro lado, a média de idade no diagnóstico do câncer de mama para mulheres brancas é 63, enquanto para as mulheres negras é 59 (Yedjou et al., 2019; Green et al., 2018).

As diferenças entre mulheres brancas e pretas também permeiam a esfera clínica do câncer de mama. A incidência de tumores triplo-negativos (carcinoma basal) em mulheres pretas, considerado o subtipo molecular mais agressivo, pode ser até o dobro da incidência em mulheres brancas, especialmente nas mais jovens. Além disso, outras diferenças de caráter biológico entre mulheres brancas e pretas podem influenciar os desfechos relacionados ao câncer de mama. Estas características envolvem fatores de crescimento, hormônios, expressão de receptores, proteínas e uma série de fatores genéticos nas populações. Entretanto, apesar dos esforços para reduzir as disparidades raciais associadas ao câncer de mama, mulheres pretas podem apresentar maior probabilidade de receberem tratamentos inapropriados, sofrerem atrasos no tempo diagnóstico e início do tratamento, além de barreiras relacionadas aos serviços e profissionais de saúde (Yedjou et al., 2019; Green et al., 2018).

Além da etnia, o estado civil de mulheres com câncer de mama pode modificar os desfechos do câncer. Uma investigação epidemiológica envolvendo o status marital, a etnia e o câncer de mama triplo-negativo concluiu que possuir um companheiro pode não influenciar nas taxas de mortalidade de mulheres pretas. Por outro lado, mulheres brancas separadas ou viúvas apresentam mais chances do que mulheres casadas para a ocorrência deste desfecho. Mulheres brancas solteiras ou divorciadas podem não se relacionar com o aumento do risco de mortalidade (Parise \& Caggiano, 2018). Em nossos resultados, $56 \%$ das mulheres com carcinoma ductal invasivo da mama possuíam companheiro, sendo $46,4 \%$ casadas.

O status socioeconômico também se relaciona com os desfechos do câncer de mama e contribui para o cenário clínicoepidemiológico atual em países em desenvolvimento. Embora a incidência de neoplasias mamárias possa ser maior em países desenvolvidos, a mortalidade pelo câncer, compreendida como o resultado de diversos componentes do tratamento oncológico, é maior em países em desenvolvimento. Dentre os fatores que compõem esse status, a baixa escolaridade é significativamente associada aos desfechos negativos no câncer de mama (Ghoncheh et al., 2015; Hortobagyi et al., 2005). Em nossos resultados, $28 \%$ das mulheres com carcinoma ductal invasivo da mama declararam desemprego quando deram entrada no serviço de oncologia, bem como 83,2\% renda familiar igual ou inferior a três salários mínimos e 19,2\% ensino superior completo.

Considerando o cenário socioeconômico, o câncer de mama pode ser associado a 27,3\% das neoplasias em mulheres que vivem em países de baixa e média renda. Neste ambiente, espera-se que até metade das mulheres com neoplasias mamárias não sobrevivam à doença e essa projeção torna-se ainda mais alarmante quando consideramos a possibilidade de mais de 30 novos casos em cada 100.000 mulheres por ano. O status socioeconômico, considerando a renda, pode interferir nos desfechos do câncer de mama em mulheres, tornando emergente a demanda de políticas públicas para diminuir as iniquidades relacionadas com a vulnerabilidade socioeconômica, especialmente em países em desenvolvimento (Dreyer et al., 2018; de Sanjose \& Tsu, 2019).

Por outro lado, as características clínico-patológicas relacionadas ao câncer de mama são importantes para compreender o comportamento destas neoplasias e os desfechos associados. O carcinoma ductal invasivo é o subtipo histológico mais comum em mulheres com neoplasia mamária invasiva, correspondendo até $84 \%$ dos carcinomas invasivos diagnosticados. O tratamento de neoplasias invasivas envolve abordagens cirúrgicas, tratamento quimioterápico e radioterapia (Hashmi et al., 2018; Akram et al., 2017).

Diversos profissionais e protocolos divergem sobre a abordagem inicial do câncer de mama, seja ele in situ ou invasivo. A remoção completa da glândula mamária em estágios avançados da doença é fortemente associada a melhores prognósticos e taxas de mortalidade por câncer de mama reduzidas. Por outro lado, cirurgias de remoção parcial são frequentemente consideradas em estágios iniciais da doença, sendo associada a outras terapias de suporte para evitar a recorrência da lesão 
tumoral. É válido salientar que, apesar de possíveis desfechos favoráveis em relação às outras abordagens, a remoção completa da mama pode estar associada à redução da qualidade de vida e alterações na autoimagem e autoestima das mulheres (Akram et al., 2017; Vanni et al., 2020). Em nossos resultados, 64,8\% das mulheres com carcinoma ductal invasivo da mama já haviam se submetido à mastectomia radical quando os dados foram coletados.

Frequentemente pacientes com câncer de mama também se beneficiam da radiação ionizante para o controle dos tumores. Com o avanço das radioterapias e a implementação de doses hipofracionadas que reduzem toxicidades radioinduzidas, mulheres com câncer de mama podem se submeter a procedimentos cirúrgicos mais conservadores de modo eficaz quando as terapias são combinadas. Especialmente em estágios iniciais da doença, como carcinomas ductais in situ, a radioterapia pode reduzir satisfatoriamente a recorrência da doença após mastectomias parciais ou totais, bem como reduzir a necessidade de mastectomias contralaterais preventivas (Akram et al., 2017; Elshof et al., 2016). Em nossos achados, apenas 36\% das mulheres com carcinoma ductal invasivo da mama receberam radioterapia até o momento da coleta dos dados.

Além disso, o tratamento quimioterápico, adjuvante ou neoadjuvante, é frequente no manejo clínico do câncer de mama. Evidências indicam que regimes de agentes antineoplásicos adjuvantes podem reduzir a recorrência e disseminação metastática do câncer de mama tratado cirurgicamente, enquanto regimes neoadjuvantes reduzem o tamanho dos tumores para torná-los cirurgicamente tratáveis, permitindo abordagens mais conservadoras para a mama (Harris et al., 2016; Masood, 2016). Por outro lado, semelhante aos nossos resultados, mulheres diagnosticadas com carcinoma ductal invasivo da mama frequentemente se expõem a procedimentos cirúrgicos avançados, como mastectomias totais, especialmente aquelas que possuem mais de 60 anos (Bao et al., 2018).

Diversas classes de agentes quimioterápicos estão disponíveis no tratamento do câncer de mama. A escolha destes agentes e seus protocolos depende de fatores que incluem o perfil molecular e histopatológico do câncer, estágio da doença, comorbidades, disponibilidade em serviços de saúde e estratégias terapêuticas selecionadas, baseando-se em evidências. As classes e medicamentos frequentemente prescritos são alquilantes (ciclofosfamida), antraciclinas (doxorrubicina e epirrubicina), taxanos (paclitaxel e docetaxel), platinas (carboplatina, cisplatina e oxaliplatina) e antimetabólitos (metotrexato) (Akram et al., 2017; Ejlertsen, 2016; Early Breast Cancer Trialists' Collaborative Group, 2002).

Ainda sim, é importante considerar os eventos adversos relacionados aos tratamentos oncológicos para o câncer de mama. A dor tem sido relatada em diversos estudos como um sintoma frequente, especialmente em mulheres que se submeteram a cirurgias na mama, pois até $60 \%$ das pacientes podem experimentar dor recorrente após mastectomias, embora a quimiorradiação também seja capaz de provocá-la. Por fim, sintomas como fadiga e alterações psíquicas, quando associados à dor, podem contribuir significativamente na redução da qualidade de vida de mulheres com câncer de mama (Wang et al., 2016; Costa et al., 2017; Reinertsen et al., 2017). Os fatores relacionados à fadiga persistente, por outro lado, também incluem alterações psíquicas como um fator preditivo para a ocorrência destas em até 10 anos após o tratamento oncológico (Reinertsen et al., 2010).

A dor e a fadiga ainda podem ser potencializadas pela existência de alterações psíquicas, especialmente a depressão. Mulheres com câncer de mama possuem alto risco de desenvolver alterações psíquicas, que são frequentemente associadas a experiências duradouras com sinais e sintomas do tratamento oncológico. A prevalência de ansiedade e/ou depressão em mulheres com câncer de mama é alta, particularmente mais elevada durante o primeiro ano após o diagnóstico, acometendo até 35,7\% destas. A angústia, sensação frequentemente relatada durante o câncer, é frequentemente subtratada ou negligenciada durante o câncer de mama, embora seja uma condição altamente prevalente e associada com outras questões de saúde mental (Tsaras et al., 2018; Rogers et al., 2017).

Fadiga, ansiedade e depressão são as principais causas de redução da qualidade de vida e incapacidade durante e após o tratamento oncológico do câncer de mama (Tsaras et al., 2018; Rogers et al., 2017). Além disso, o surgimento e a persistência 
de alterações psíquicas estão fortemente associados à excisão cirúrgica da mama durante o tratamento. Apesar de não haver uma heterogeneidade na percepção feminina sobre a importância das mamas nas relações sociais e sexuais, o medo de perdê-las e o impacto provocado pela perda podem desencadear alterações na autoimagem e autoestima, comprometendo a qualidade de vida e a sexualidade durante e após o tratamento oncológico (Zhang et al., 2015; Telli \& Gürkan, 2019; Cieślak \& Golusiński, 2018).

No que se refere às comorbidades, doenças cardiovasculares e o câncer de mama compartilham de diversos fatores de risco, como idade, tabagismo, dieta, obesidade e sedentarismo. Por outro lado, a preexistência de doenças cardiovasculares pode interferir na terapêutica oncológica, bem como diversos agentes quimioterápicos podem apresentar cardiotoxicidade. Estes fenômenos estreitam as relações entre o câncer de mama e o adoecimento cardiovascular, considerando que doenças cardiovasculares também são as principais causas de morte em mulheres após o tratamento oncológico (Mehta et al., 2018; Haque et al., 2014). Especificamente sobre a hipertensão arterial sistêmica, as atuais evidências indicam que esta comorbidade aumenta o risco de câncer de mama, especialmente em mulheres hipertensas na pré-menopausa (Han et al., 2017).

Para atender às demandas provocadas pelo câncer, sistemas e serviços de saúde precisam de estratégias eficientes para o gerenciamento dos cuidados. Considerando a complexidade do manejo de neoplasias, mecanismos de troca de informação e linhas de cuidados consolidadas permitem a construção e manutenção de serviços oncológicos satisfatórios para pacientes, profissionais e gestores dentro de um sistema de saúde (van Hoeve et al., 2018; Fennell et al., 2010). A articulação entre pacientes, profissionais e serviço de oncologia deve ser pautada com o rigor e qualificação inerentes ao cuidado humano, fornecendo aos pacientes o suprimento de suas necessidades ao passo que disponibiliza todas as redes de suporte necessárias para o enfrentamento do câncer (Kullberg et al., 2015; Hahlweg et al., 2017).

No Brasil, diversos outros estudos buscaram compreender o perfil clínico e/ou epidemiológico do câncer de mama das mulheres brasileiras. Nas regiões Norte e Nordeste, os estudos de Lôbo et al. (2020) e Oliveira et al. (2018) verificaram a frequência de mulheres não-brancas em torno de 50\%, onde mulheres pretas e pardas eram maioria. Estes resultados divergem de estudos realizados por Souza et al. (2018), Santos et al. (2019) e Lena et al. (2019), realizados nas regiões Sudeste e Sul, onde a maioria das mulheres diagnosticadas com câncer de mama eram brancas (94\%, 74,1\% e 93,7\%, respectivamente). Os nossos resultados convergem com os dados norte-nordestinos, apresentando $67,6 \%$ de mulheres pardas ou pretas com carcinoma ductal invasivo da mama.

O núcleo familiar é outro fator que demonstra relevância na temática do câncer de mama em outras investigações epidemiológicas. O estudo internacional publicado por Parise e Caggiano (2018) identificou que a situação matrimonial pode ser um fator de risco para mortalidade pelo câncer de mama, concluindo que mulheres casadas podem apresentar melhores taxas de sobrevida quando comparadas às divorciadas ou viúvas. Nos nossos resultados, observamos que $46,4 \%$ eram mulheres casadas e 44\% não possuíam companheiro no momento em que os dados foram coletados.

O ensino fundamental, completo ou não, é o nível de escolaridade da maioria das mulheres diagnosticadas em todo o Brasil com câncer de mama, conforme demonstram os resultados de Rocha et al. (2018), Oliveira et al. (2018) e Magalhães et al. (2017). Em diversos estudos, a baixa escolaridade pode ser considerada um fator de risco importante para a mortalidade por câncer de mama. Nas amostras estudadas pelos autores, $0,3 \%$ a 8,77\% de mulheres com câncer de mama afirmaram ter cursado ensino superior, prevalecendo os níveis de escolaridade do ensino fundamental e médio. Em nossos resultados, 33,6\% das mulheres possuíam ensino fundamental e 36\% ensino médio, corroborando com a prevalência destes níveis de escolaridade em mulheres com câncer de mama.

A escolaridade frequentemente se correlaciona com a renda familiar média. Um estudo brasileiro concluiu que a renda familiar também é um fator de risco para o câncer de mama, pois, quando associado à escolaridade das mulheres, é possível observar um maior nível de desinformação sobre comportamentos e métodos de prevenção para o câncer de mama (Gonçalves et al., 2017). Em nossos resultados, a renda mensal familiar entre 1 e 3 salários mínimos foi a mais frequente (77,6\%). 
Das mulheres diagnosticadas com carcinoma ductal invasivo, de acordo com Souza et al. (2018) e Rocha et al. (2018), mais de $90 \%$ das pacientes são tratadas com sessões de quimioterapia, reportando que os protocolos à base de antraciclina (doxorrubicina) esteve presente em 62,6\% dos casos, bem como doxorrubicina combinada com ciclofosfamida e docetaxel (TAC) em 74\% dos casos, respectivamente (Souza et al., 2018; Rocha et al., 2018). Em nossos resultados, 59,2\% das pacientes se submeteram à combinação de ciclofosfamida e doxorrubicina.

A combinação com radioterapia e intervenções cirúrgicas também estão presentes na literatura e podem ser consideradas abordagens eficazes para o câncer de mama. Um estudo do Instituto Nacional do Câncer (INCA), realizado por Souza et al. (2018), verificou que 94,5\% das pacientes se submeteram à mastectomia e 76\% à radioterapia. Em nossos resultados, 86,4\% das pacientes se submeteram a algum tipo de mastectomia (parcial ou total).

Considerando as alterações psíquicas, o estudo brasileiro de Salvetti et al. (2020) aponta para uma correlação significativa entre a depressão com o sintoma da fadiga em pacientes em tratamento oncológico com quimioterapia e radioterapia. Com base na escala de qualidade de vida da European Organization for Research and Treatment of Cancer Core Quality of Life Questionnaire (EORTC QLQ-C30), os autores puderam concluir que a fadiga (76,6\%), a insônia (47,7\%) e a dor (42,1\%) foram as principais alterações identificadas, seguidos de perda de apetite $(37,4 \%)$. Por outro lado, não foi observada uma associação significativa entre depressão e dor. Esses resultados relacionam-se com os achados de Imran et al. (2019), que relatam os mesmos sintomas frequentes em sua amostra, com inclusão da inapetência em 284 mulheres com câncer de mama na Arábia Saudita, cujas frequências foram: insônia (31,3\%), fadiga (28,2\%), dor (34,5\%) e perda de apetite (41,9\%). A ocorrência desses sintomas também foi frequente em nossos resultados, especialmente dor $(83,2 \%)$ e a fadiga $(68,8 \%)$.

Além disso, outros três estudos brasileiros se propuseram a descrever o perfil epidemiológico das mulheres com câncer de mama em seus respectivos estados: Minas Gerais, Pará e Piauí. Verificando os resultados, observa-se divergências em relação às comorbidades mais frequentes. Gonçalves et al. (2018) mostra que em Minas Gerais a hipertensão arterial sistêmica predominou (42,5\%), seguida da diabetes mellitus (16,4\%). Por outro lado, Rocha et al. (2018) e Oliveira et al. (2018) trazem o sobrepeso e a obesidade grau 1 como as comorbidades mais frequentes e preocupantes em mais da metade das amostras estudadas (Oliveira et al., 2018; Rocha et al., 2018). Em nossos resultados, a hipertensão arterial sistêmica foi a mais frequente (40\%), seguida por artrite/artrose $(18,4 \%)$. A diabetes mellitus foi a quarta comorbidade mais frequente $(11,2 \%)$.

É importante informar os leitores sobre os prováveis vieses desta investigação para que sejam considerados previamente ao uso e interpretação dos resultados apresentados. É importante ressaltar que não houve nenhum treinamento dos profissionais do serviço de saúde estudado para o preenchimento das informações contidas nos prontuários clínicos. Além disso, considerando que um único serviço de saúde foi escolhido e avaliado pelos pesquisadores, bem como não foram feitas estimativas amostrais, a validade interna deve ser cautelosa.

\section{Conclusão}

Podemos concluir que o perfil clínico-epidemiológico das mulheres com carcinoma ductal invasivo da mama verificado na amostra apresentou ampla variação de componentes sociodemográficos e clínicos, sendo esses relevantes para a saúde, bemestar e qualidade de vida destas pacientes, indicando que a compreensão destes perfis pode auxiliar no enfrentamento do câncer e seus impactos biopsicossociais.

\section{Referências}

Akram, M., Iqbal, M., Daniyal, M. \& Khan, A. U. (2017). Awareness and current knowledge of breast cancer. Biol Res, $50(1), 33$.

Anampa, J., Makower, D. \& Sparano, J. A. (2015). Progress in adjuvant chemotherapy for breast cancer: an overview. BMC Med, 17 (13), 195. 
Arps, D. P., Healy, P., Zhao, L., Kleer, C. G. \& Pang, J. C. (2013). Invasive ductal carcinoma with lobular features: a comparison study to invasive ductal and invasive lobular carcinomas of the breast. Breast Cancer Res Treat, 138(3), 719-26.

Bao, X., Sun, K., Tian, X., Yin, Q., Jin, M., Yu, N., Jiang, H., Zhang, J. \& Hu, Y. (2018). Present and changing trends in surgical modalities and neoadjuvant chemotherapy administration for female breast cancer in Beijing, China: A 10-year (2006-2015) retrospective hospitalization summary report-based study. Thorac Cancer, 9(6), 707-717.

Cabral, A. L. L.V., Giatti, L., Casale, C. \& Cherchiglia, M. L. (2019). Social vulnerability and breast cancer: differentials in the interval between diagnosis and treatment of women with different sociodemographic profiles. Cien Saude Colet, 24(2), 613-622.

Casasent, A. K., Schalck, A., Gao, R., Sei, E., Long, A., Pangburn, W., Casasent, T., Bernstam, M. F., Edgerton, M. E. \& Navin, N. E. (2018). Multiclonal Invasion in Breast Tumors Identified by Topographic Single Cell Sequencing. Cell, 172(1-2), 205-217.

Cieślak, K. \& Golusiński, W. (2018). Coping with loss of ability vs. emotional control and self-esteem in women after mastectomy. Rep Pract Oncol Radiothe, 23(3), 168-174.

Costa, W. A., Monteiro, M. N., Queiroz, J. F. \& Gonçalves, A. K. (2017). Pain and quality of life in breast cancer patients. Clinics (Sao Paulo), 72(12), 758763.

de Sanjose, S. \& Tsu, V. D. (2019). Prevention of cervical and breast cancer mortality in low- and middle-income countries: a window of opportunity. Int J Womens Health, 11:381-386.

Dreyer, M. S., Nattinger, A. B., McGinley, E. L. \& Pezzin, L. E. (2018). Socioeconomic status and breast cancer treatment. Breast Cancer Res Treat, 167(1), 18.

Early Breast Cancer Trialists' Collaborative Group (2002). Multi-agent chemotherapy for early breast cancer. Cochrane Database Syst Rev, (1), CD000487.

Ejlertsen, B. (2016) Adjuvant chemotherapy in early breast cancer. Dan Med J, 63(5), B5222.

Elshof, L. E., Schaapveld, M., Schmidt, M. K., Rutgers, E. J., Van, L. F. E. \& Wesseling, J. (2016). Subsequent risk of ipsilateral and contralateral invasive breast cancer after treatment for ductal carcinoma in situ: incidence and the effect of radiotherapy in a population-based cohort of 10,090 women. Breast Cancer Res Treat, 159(3), 553-63.

Fennell, M. L., Das, I. P., Clauser, S., Petrelli, N. \& Salner, A. (2010). The organization of multidisciplinary care teams: modeling internal and external influences on cancer care quality. J Natl Cancer Inst Monogr, 2010(40), 72-80.

Ghoncheh, M., Mirzaei, M, \& Salehiniya, H. (2015). Incidence and Mortality of Breast Cancer and their Relationship with the Human Development Index (HDI) in the World in 2012. Asian Pac J Cancer Prev, 16(18), 8439-8443.

Gonçalves, C. V., Camargo, V. P., Cagol, J. M., Miranda, B. \& Mendoza, R. A. S. (2017). O conhecimento de mulheres sobre os métodos para prevenção secundária do câncer de mama. Ciênc saúde coletiva, 22(12), 4073-4081.

Gonçalves, M. M., Guedes, N. A. B., Matos, S. S., Tiensoli, S. D., Simino, G. P. R. \& Corrêa, A. R. (2018). Perfil dos Atendimentos a Pacientes Oncológicos em uma Unidade de Pronto Atendimento. Rev. enferm. Cent.-Oeste Min, 8, e2595.

Green, A. K., Aviki, E. M., Matsoukas, K., Patil, S., Korenstein, D. \& Blinder, V. (2018). Racial disparities in chemotherapy administration for early-stage breast cancer: a systematic review and meta-analysis. Breast Cancer Res Treat, 172(2), 247-263.

Hahlweg, P., Didi, S., Kriston, L., Härter, M., Nestoriuc, Y. \& Scholl, I. (2017). Process quality of decision-making in multidisciplinary cancer team meetings: a structured observational study. BMC Cancer, 17(1),772.

Han, H., Guo, W., Shi, W., Yu, Y., Zhang, Y., Ye, X. \& He, J. (2017). Hypertension and breast cancer risk: a systematic review and meta-analysis. Sci Rep, 7:44877.

Haque, R., Prout, M., Geiger, A. M., Kamineni, A., Thwin, S. S. \& Avila, C. (2014). Comorbidities and cardiovascular disease risk in older breast cancer survivors. Am J Manag Care, 20(1), 86-92.

Harris, L. N., Ismaila, N., McShane, L. M., Andre, F., Collyar, D. E., Gonzalez, A., Hammond, E. H., Kuderer, N. M., Liu, M. C., Mennel, R. G., Ponazak, C. V., Bast, R. C. \& Hayes, D. F. (2016). Use of Biomarkers to Guide Decisions on Adjuvant Systemic Therapy for Women With Early-Stage Invasive Breast Cancer: American Society of Clinical Oncology Clinical Practice Guideline. J Clin Oncol, 34(10), 1134-50.

Hashmi, A. A., Aijaz, S., Mahboob, R., Khan, S. M., Irfan, M., Iftikhar, N., Nisar, M., Siddiqui, M., Edhi, M. M., Faridi, N. \& Khan, A. (2018). Clinicopathologic features of invasive metaplastic and micropapillary breast carcinoma: comparison with invasive ductal carcinoma of breast. BMC Res Notes, 11(1), 531.

Hortobagyi, G. N., de la Garza, S. J., Pritchard, K., Amadori, D., Haidinger, R., Hudis, C.A., Khaled, H., Liu, M. C., Martin, M., Namer, M., O'Shaughnessy, J. A., Shen, Z. Z. \& Albain, K. S. (2005). The global breast cancer burden: variations in epidemiology and survival. Clin Breast Cancer, 6(5), 391-401.

Imran, M., Al-Wassia, R., Alkhayyat, S. S., Baig, M. \& Al-Saati, B. A. (2019). Assessment of quality of life (QoL) in breast cancer patients by using EORTC QLQ-C30 and BR-23 questionnaires: A tertiary care center survey in the western region of Saudi Arabia. PLoS One, 14(7), e0219093.

Kullberg, A., Sharp, L., Johansson, H. \& Bergenmar, M. (2015). Information exchange in oncological inpatient care--patient satisfaction, participation, and safety. Eur J Oncol Nurs, 19(2), 142-147. 
Lena, P. T., Rempel, C., Coltro, D., Frigeri, C. D. L. \& Grave, M. T. Q. (2019). Perfil epidemiológico de mulheres mastectomizadas em um serviço de referência localizado no Vale do Taquari/RS. Rev epidemiol controle infecç, 9(2),122-128.

Li, C. I., Uribe, D. J. \& Daling, J. R. (2005). Clinical characteristics of different histologic types of breast cancer. Br J Cancer, 31, 93(9), 1046-52.

Lôbo, J. L. S., Silva, M. L. C., Tomé, T. K. B. V. \& de Souza, C. D. F. (2020). Mortalidade por Câncer de Mama Feminino em Alagoas no Período de 2001 a 2016: Análise de Tendência e Distribuição Espacial. Rev bras cancerol, 66(1), e-09656.

Magalhães, G., Brandão, S. C., Fustinoni, S. M., Matos, J. C. \& Schirmer, J. (2017). Perfil clínico, sociodemográfico e epidemiológico da mulher com câncer de mama. J. res.: fundam. care. online, $9(2), 473-479$.

Masood, S. (2016). Neoadjuvant chemotherapy in breast cancers. Women's Health (Lond), 12(5), 480-491.

Mathew, A., George, P. S., Ramadas, K., Mathew, B. S., Kumar, A., Roshni, S., Jayakumar, K. N. L \& Booth, C. M. (2019). Sociodemographic Factors and Stage of Cancer at Diagnosis: A Population-Based Study in South India. J Glob Oncol. 5, 1-10.

Mehta, L. S., Watson, K. E., Barac, A., Beckie, T. M., Bittner, V., Cruz, S. F., Dent, S., Kondapalli, L., Ky, B., Okwuosa, T., Piña, L. I. \& Volgman, A. S. (2018). Cardiovascular Disease and Breast Cancer: Where These Entities Intersect: A Scientific Statement From the American Heart Association. Circulation, 137(8), e30-e66.

Moo, T. A., Sanford, R., Dang, C. \& Morrow, M. (2018). Overview of Breast Cancer Therapy. PET Clin, 13(3), 339-354.

Oliveira, V. A. S., Vilarinho, M. L. C. M. \& Milanez, L. L. S. (2018). Characterization of women at risk of breast cancer at Primary Health Care. Rev Enferm UFPI, 7(1), 38-43.

Parise, C. \& Caggiano, V. (2018). The influence of marital status and race/ethnicity on risk of mortality for triple negative breast cancer. PLoS One, 13(4).

Reinertsen, K. V., Cvancarova, M., Loge, J. H., Edvardsen, H., Wist, E. \& Fosså, S. D. (2010). Predictors and course of chronic fatigue in long-term breast cancer survivors. J Cancer Surviv, 4(4), 405-14.

Reinertsen, K. V., Engebraaten, O., Loge, J. H., Cvancarova, M., Naume, B., Wist, E., Edvardsen, H., Wille, E., Bjøro, T. \& Kiserud, C. E. (2017). Fatigue During and After Breast Cancer Therapy-A Prospective Study. J Pain Symptom Manage, 53(3), 551-560.

Rocha, F. S., Silva, W. S., Nascimento, E. R. \& Bacciotti, A. M. (2018). Epidemiological profile of breast câncer in a reference hospital in the north region. Mastology, 28(3), 169-75.

Rogers, L. Q., Courneya, K. S., Anton, P. M., Verhulst, S., Vicari, S. K., Robbs, R. S. \& McAuley, E. (2017). Effects of a multicomponent physical activity behavior change intervention on fatigue, anxiety, and depressive symptomatology in breast cancer survivors: randomized trial. Psychooncology, 26(11), 19011906.

Salvetti, M. G., Machado, C. S. P., Donato, S. C. T. \& Silva, A. M. (2020). Prevalência de sintomas e qualidade de vida de pacientes com câncer. Rev bras enferm, 73(2), e20180287.

Santos, J. C. M., Silva, C. M., Teixeira, J. J. V. \& Peder, L. D. (2019). Perfil epidemiológico e clínico de mulheres com câncer de mama na região oeste do Paraná. Rev bras ciênc saúde, 23(4), 449-458.

Souza, J. C. S. O., Nunes, A. S., Silva, J. L., Gonçalves, A. C. \& Costa, S. C. V. (2018). Real-World Data Related to Non-Metastatic Breast Cancer in Young Women: Experience of a Single Institution. Rev bras cancerol, 64(1), 45-53.

Sun, Y. S., Zhao, Z., Yang, Z. N., Xu, F., Lu, H. J., Zhu, Z. Y., Shi, W., Jiang, J., Yao, P. P., \& Zhu, H. P. (2017). Risk Factors and Preventions of Breast Cancer. Int J Biol Sci, 13(11), 1387-1397.

Telli, S. \& Gürkan, A. (2019). Examination of Sexual Quality of Life and Dyadic Adjustment among Women with Mastectomy. Eur J Breast Health, 16(1), 4854 .

Tsaras, K., Papathanasiou, I. V., Mitsi, D., Veneti, A., Kelesi, M., Zyg, S. \& Fradelos, E. C. (2018). Assessment of Depression and Anxiety in Breast Cancer Patients: Prevalence and Associated Factors. Asian Pac J Cancer Prev, 25.,19(6), 1661-1669.

van Hoeve J. C., Vernooij, R. W. M., Lawal, A. K., Fiander, M., Nieboer, P., Siesling, S. \& Rotter, T. (2018). Effects of oncological care pathways in primary and secondary care on patient, professional, and health systems outcomes: protocol for a systematic review and meta-analysis. Syst Ver, 7(1), 49.

Vanni, G., Materazzo, M., Pellicciaro, M., Morando, L., Portarena, I., Anemona, L., D'Angelillo, M. R., Barbarino, R., Chiaravalloti, A., Meucci, R., Perretta, T., Deiana, C., Orsaria, P., Caspi, J., Pistolese, C. A. \& Buonomo, O. C. (2020). Does Age Matter? Estimating Risks of Locoregional Recurrence After Breastconservative Surgery. In Vivo, 34(3), 1125-1132.

Wang, L., Guyatt, G. H., Kennedy, S. A., Romerosa, B., Kwon, H. Y., Kaushal, A., Chang, Y., Craigie, S., de Almeida, C. P. B., Couban, R. J., Parascandalo, S. R., Izhar, Z., Reid, S., Khan, J. S., McGillion, M. \& Busse, J. W. (2016). Predictors of persistent pain after breast cancer surgery: a systematic review and metaanalysis of observational studies. CMAJ, 188(14), E352-E361.

Weigelt, B., Geyer, F. C. \& Reis, F. J. S. (2010). Histological types of breast cancer: how special are they? Mol Oncol, 4(3), 192-208.

Yedjou, C. G., Sims, J. N., Miele, L., Noubissi, L., Lowe, L., Fonseca, D. D., Alo, R. A., Payton, M. \& Tchounwou, P. B. (2018). Health and Racial Disparity in Breast Cancer. Adv Exp Med Biol, 1152:31-49.

Yeo, S. K. \& Guan, J. L. (2017). Breast Cancer: Multiple Subtypes within a Tumor? Trends Cancer, 3(11). 753-760. 
Research, Society and Development, v. 10, n. 1, e39110111836, 2021

(CC BY 4.0) | ISSN 2525-3409 | DOI: http://dx.doi.org/10.33448/rsd-v10i1.11836

Zhang, Y., Xu, H., Wang, T., He, J., Qiao, Y., Wei, J. \& Dong, J. (2015). Psychosocial Predictors and Outcomes of Delayed Breast Reconstruction in Mastectomized Women in Mainland China: An Observational Study. PLoS One, 10(12), e0144410. 